\title{
Repercussão da doença arterial periférica na tolerância ao exercício e na qualidade de vida de idosos e o papel da fisioterapia cardiovascular: artigo de revisão
}

\author{
The impact of peripheral arterial disease on exercise tolerance and quality of life in the \\ elderly and the role of cardiovascular physiotherapy: review article
}

Ana Leticia Gonçalves Lourenço ${ }^{1}$, Josicléia Leôncio da Silva ${ }^{1}$ (D) , Jéssica Costa Leite

\begin{abstract}
Resumo
A doença arterial periférica tem como principal sintoma a claudicação intermitente, fator que resulta em incapacidade funcional, comprometendo a qualidade de vida. Esta revisão objetivou fazer um levantamento sobre os impactos ocasionado pela doença nos idosos, investigando as possíveis contribuições da fisioterapia cardiovascular. Para isso, realizou-se uma busca nas bases de dados MEDLINE, LILACS, SciELO, Scopus, Science Direct e PEDro, a qual identificou 7.587 estudos. Desses, sete atenderam aos critérios de elegibilidade, sendo então agrupados e analisados conforme o nível de evidência, grau de recomendação e qualidade metodológica. Pôde-se observar um considerável impacto da doença sobre a tolerância ao exercício e a qualidade de vida. Quanto à abordagem terapêutica desses desfechos, os estudos relataram que houve melhora na deambulação e na qualidade de vida, aumento da capacidade funcional e redução da dor. No que se refere à modalidade de tratamento, a maioria das pesquisas incluíram exercícios aeróbicos.
\end{abstract}

Palavras-chave: doença arterial periférica; tolerância ao exercício; qualidade de vida.

\begin{abstract}
The primary symptom of peripheral arterial its intermittent claudication; a condition that causes functional disabilities, compromising quality of life. This review aimed to survey the impacts of this disease on the elderly, investigating possible contributions that cardiovascular physiotherapy has to offer. Searches were run on the MEDLINE, LILACS, SciELO, Scopus, Science Direct, and PEDro databases, identifying 7,587 studies. Seven of these met the eligibility criteria and were grouped and analyzed according evidence level, recommendation grade, and methodological quality. It was observed that this disease is responsible for considerable impact on exercise tolerance and quality of life. Regarding the therapeutic approach to these outcomes, the studies reported that there were improvements in walking and quality of life, increased functional capacity, and reduced pain. With regard to the treatment modality, most research included aerobic exercises.
\end{abstract}

Keywords: peripheral arterial disease; exercise tolerance; quality of life.

Como citar: Lourenço ALG, Silva JL, Leite JC. Repercussão da doença arterial periférica na tolerância ao exercício e na qualidade de vida de idosos e o papel da fisioterapia cardiovascular: artigo de revisão. J Vasc Bras. 2021;20:e20200117. https://doi.org/10.1590/1677-5449.200117 


\section{INTRODUÇÃO}

A doença arterial obstrutiva periférica pode causar obstrução parcial ou total das artérias dos membros inferiores, provocando redução do fluxo sanguíneo para as extremidades. Nos casos mais críticos, existe o risco de amputação do membro, caso não haja possibilidade de revascularização $0^{1,2}$.

Aproximadamente $20 \%$ da população acometida pela doença têm mais de 65 anos de idade. No Brasil, estima-se que anualmente $0,053 \%$ da população seja diagnosticada com doença arterial periférica (DAP), incluindo homens com idade entre 55 e 74 anos e mulheres de 65 a 74 anos. Essa doença pode progredir de forma assintomática em até $80 \%$ dos casos, o que atrasa e dificulta o diagnóstico precoce, comprometendo o prognóstico ${ }^{3,4}$.

Dentre os principais fatores de risco para DAP, destacam-se: diabetes, tabagismo, hipertensão e dislipidemia, condições preponderantes na população idosa ${ }^{5}$. Quanto à apresentação clínica, destaca-se a claudicação intermitente, a qual acomete cerca de um terço dos pacientes. Além disso, são frequentes sintomas como cãibra, dor ou cansaço nos membros inferiores (MMII), que pioram no decorrer da caminhada e aliviam no repouso ${ }^{6}$.

Em estágios mais avançados da DAP, pode ocorrer necrose tecidual, aumentando significativamente o risco de o indivíduo perder o membro acometido ${ }^{6}$. Todos esses fatores fazem com que a DAP seja responsável pela redução da capacidade funcional e pela piora da qualidade de vida $(\mathrm{QV})$ dos pacientes ${ }^{1,3}$.

Dessa forma, é recomendada a avaliação vascular precoce nesses indivíduos, a fim de que se determine a capacidade e a tolerância ao exercício. Para isso, podem ser utilizadas medidas diretas, por meio do teste de esteira ou do teste de caminhada de seis minutos (TC6), assim como medidas indiretas, utilizando escalas específicas ${ }^{7}$. Já a QV pode ser avaliada por questionários que consideram tanto aspectos físicos, psicológicos e sociais como a percepção do indivíduo ${ }^{8}$.

Nesse contexto, a fisioterapia apresenta um importante papel no tratamento da DAP, pois pode atuar no pré-operatório com o objetivo de controlar a dor, reduzir edemas, aumentar a amplitude de movimento, estimular a deambulação e propiciar a educação em saúde; e no pós-operatório com o objetivo de promover ganho de força muscular, amplitude de movimento e capacidade funcional, por meio de exercícios resistidos, aeróbicos e de flexibilidade ${ }^{2}$.

Diante do exposto, considerando o crescente número de idosos acometidos pela DAP no Brasil, pesquisar sobre essa temática, em busca de informação acerca das terapêuticas existentes e das alterações relacionadas à doença, pode elevar as chances de sucesso no tratamento. Portanto, este trabalho se propôs a revisar a literatura a fim de verificar o impacto da DAP na tolerância ao exercício e na QV de idosos, além de investigar as possíveis contribuições da fisioterapia para minimizar essas alterações.

\section{MÉTODOS}

Trata-se de uma revisão integrativa da literatura, em que se realizou uma busca por artigos indexados nas bases de dados MEDLINE/Pubmed, LILACS, SciELO, Scopus (Elsevier), Science Direct e PEDro. Foi realizada também a busca manual nas referências dos artigos incluídos nesta pesquisa e das revisões integrativas já existentes sobre essa temática, em busca de quaisquer outros estudos potencialmente elegíveis.

Incluíram-se na pesquisa artigos em qualquer idioma, sem restrição de ano, com todos os tipos de delineamento, que avaliaram pacientes idosos com DAP e/ou que realizaram atendimento fisioterapêutico. Excluíram-se artigos de revisão, estudos em que a intervenção tinha sido interrompida ou com resultados inconclusivos, e protocolos de estudos futuros.

O levantamento foi realizado em abril e maio de 2020, e todas as buscas foram executadas em inglês. Utilizaram-se os operadores booleanos "AND" e "OR" para montar as estratégias de busca, que foram adaptadas conforme as exigências de cada base. Os descritores MeSH (Medical Subject Headings) adotados foram: "peripheral arterial disease", "peripheral artery disease", "exercise tolerance", "elderly", "quality of life", "HRQOL", "health related quality of life", "life quality", "physical therapy specialty", "physiotherapy specialty" e "physiotherapy".

A questão de pesquisa foi fundamentada por meio da estratégia PICO, na qual "P" refere-se ao paciente ou problema (idosos com DAP); "I" à intervenção estudada (fisioterapia); "C" à comparação ou controle (não foi considerado neste estudo); e "O" ao desfecho de interesse (tolerância ao exercício e QV). Portanto, a pergunta norteadora para a condução da presente pesquisa foi: “A DAP traz repercussões negativas na tolerância ao exercício e na QV dos idosos? De que forma a fisioterapia pode auxiliar na melhora dessas alterações?".

Para o processamento e análise dos dados, foi utilizada a escala Oxford Centre for Evidence-based Medicine, a fim de avaliar o nível de evidência e o grau de recomendação por tipo de pesquisa. Já para avaliar os estudos selecionados que se caracterizaram como ensaios clínicos, foi utilizada a escala PEDro, a qual avalia estudos controlados aleatorizados em termos de qualidade metodológica, validade interna e descrição estatística. 


\section{RESULTADOS}

Foram identificados 7.587 estudos, obtidos nos seguintes bancos de dados: MEDLINE (584), LILACS (1.873), SciELO (1.116), Scopus (1.603), PEDro (44) e Science Direct (2.367). Após a aplicação dos critérios de elegibilidade, sete artigos foram selecionados para compor esta pesquisa. $\mathrm{O}$ esquema dos resultados das buscas está representado na Figura 1.

Quanto às características dos estudos selecionados, $71,44 \%$ eram ensaios clínicos controlados ${ }^{9-13}, 14,3 \%$ eram ensaios clínicos não controlados ${ }^{14}$, e 14,3\% eram estudos transversais do tipo caso-controle ${ }^{15}$. Ao todo, 685 pacientes fizeram parte dos estudos analisados, em sua maioria indivíduos do sexo masculino $(77,7 \%)$. Uma síntese dos principais achados está representada na Tabela 1.

No que se diz respeito à qualidade metodológica, $85,72 \%$ dos artigos preencheram os requisitos para avaliação, conforme os critérios da escala PEDro, à exceção de um estudo ${ }^{15}$, por se tratar de uma pesquisa de caráter transversal do tipo caso-controle. Após a avaliação, evidenciou-se que $71,44 \%$ das pesquisas exibiram escores medianos de qualidade metodológica, e $28,56 \%$ exibiram escore baixo, considerando que a pontuação máxima é 10. O grau de recomendação dos artigos foi alto, indicando que os estudos selecionados apresentaram um elevado grau de confiança e recomendação. Os níveis de evidência, grau de recomendação e qualidade metodológica de cada estudo estão expostos na Tabela 2.

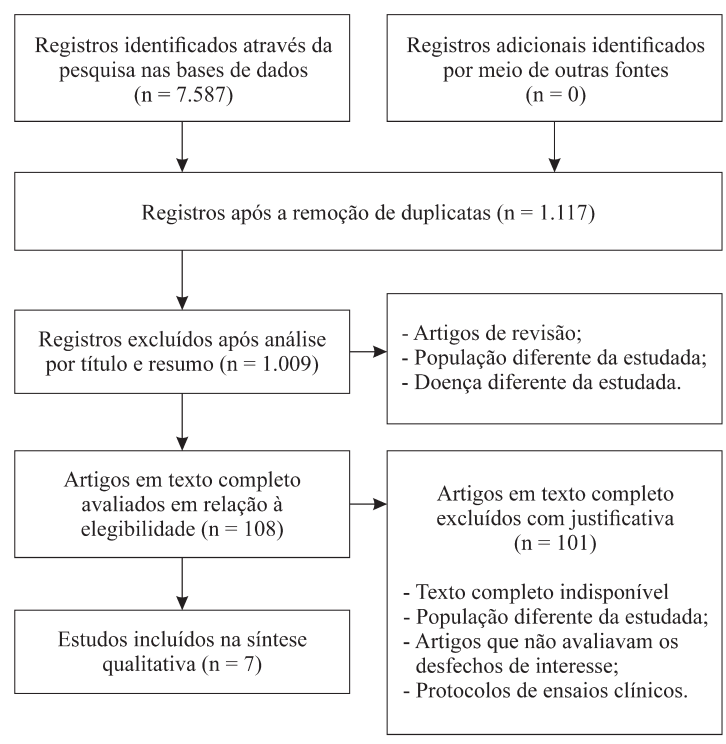

Figura 1. Fluxograma do processo de busca e seleção dos artigos.

\section{DISCUSSÃO}

Ao analisar os protocolos de tratamento, evidenciouse uma diversidade nas abordagens utilizadas. Alguns estudos, como os de Langbein et al. ${ }^{9}$ e Collins et al. ${ }^{11}$, adotaram caminhadas nórdicas visando potencializar a tolerância ao exercício, embora divergindo quanto à periodicidade e à duração da intervenção. Essa modalidade de caminhada é realizada apoiando-se em dois bastões, o que reduz o impacto nos MMII ao mesmo tempo em que permite a movimentação mais intensa dos membros superiores (MMSS) e do tronco.

Já as pesquisas de Lamberti et al. ${ }^{12}$ e Malagoni et al. ${ }^{14}$ utilizaram sessões intermitentes de caminhadas tradicionais. O grupo controle formado por Lamberti et al. ${ }^{12}$ era composto por pacientes submetidos a cirurgia de revascularização. Por fim, Tew et al. ${ }^{10} \mathrm{e}$ Akerman et al. ${ }^{13}$ divergiram totalmente dos anteriores, pois a intervenção usada consistia em exercícios aeróbicos de MMSS, imersões em água quente, exercícios resistidos e calistênicos.

Quanto às ferramentas de avaliação, houve semelhanças. Em geral, os indivíduos foram analisados pelo questionário Medical Outcomes Short-Form Health Survey (SF-36), a fim de mensurar a QV, em pelo menos $57,14 \%$ dos estudos. Já o desfecho tolerância ao exercício foi mensurado em todas as pesquisas. Para isso, foram utilizados os seguintes instrumentos: Walking Impairment Questionnaire, testes de esteira com carga incremental e constante, teste em ergômetro de MMSS, TC6 e análise do consumo máximo de oxigênio $\left(\mathrm{VO}_{2} \max \right)$.

No que se refere à avaliação da perfusão de oxigênio nos tecidos e do aumento ou diminuição de temperatura corporal promovidos pela intervenção proposta, os estudos adotaram os seguintes métodos de mensuração: espectroscopia no infravermelho próximo, dilatação mediada por fluxo, velocidade de onda de pulso, pletismografia de oclusão venosa, reinalação de monóxido de carbono e cápsula de telemetria.

\section{Impacto da DAP na tolerância ao exercício de idosos e o papel da fisioterapia}

O estudo de Dziubek et al. ${ }^{15}(\mathrm{n}=135)$ avaliou a tolerância ao exercício e a força dos músculos dos MMII em idosos saudáveis e com DAP. Os autores concluíram que a DAP reduz a tolerância ao exercício e a capacidade funcional, devido à diminuição significativa da força e da velocidade de contração muscular $(\mathrm{p}<0,005)$, da distância para claudicação absoluta $(\mathrm{p}<0,0001)$, da distância percorrida em metros $(\mathrm{p}<0,01)$ e da velocidade da marcha $(\mathrm{p}<0,01)$. 
Tabela 1. Caracterização dos estudos selecionados.

\begin{tabular}{|c|c|c|c|c|}
\hline Autor, país & Tipo de estudo & População & Avaliação & Intervenção \\
\hline $\begin{array}{l}\text { Langbein et al. }{ }^{9}, \\
\text { EUA }\end{array}$ & ECR controlado & $\begin{array}{l}\mathrm{n}=52(51 \mathrm{H} \text { e } 1 \mathrm{M}) \\
\text { média de } 67,05 \pm 7,95 \\
\text { anos }\end{array}$ & $\begin{array}{c}\text { Tolerância ao exercício: teste de } \\
\text { esteira com carga incremental, } \\
\text { carga constante e análise do } \\
\qquad \mathrm{VO}_{2} \max \end{array}$ & $\begin{array}{l}\text { G1: caminhada nórdica } 3 x \text { por } \\
\text { semana durante } 24 \text { semanas. } \\
\text { G2: grupo controle, sem exer- } \\
\text { cícios. }\end{array}$ \\
\hline $\begin{array}{l}\text { Tew et al. } .^{10} \text {, } \\
\text { Reino Unido }\end{array}$ & ECR controlado & $\begin{array}{l}\mathrm{n}=57(57 \mathrm{H} \mathrm{e} 0 \mathrm{M}) \\
\text { média de } 70 \pm 8 \text { anos }\end{array}$ & $\begin{array}{l}\text { Tolerância ao exercício: teste } \\
\text { em cicloergômetro com freio } \\
\text { eletrônico, manivela de braço, } \\
\text { análise do } \mathrm{VO}_{2} \text { max concentra- } \\
\text { ção de lactato no sangue e teste } \\
\text { incremental em esteira }\end{array}$ & $\begin{array}{l}\text { G1: exercício aeróbico intermi- } \\
\text { tente ( } 2 \text { min de exercício e } 2 \\
\text { de repouso) em ergômetro de } \\
\text { MMSS, } 2 x \text { por semana, de } 20 \text { a } 40 \\
\text { min por dia, durante } 12 \text { semanas. } \\
\text { G2: grupo controle, sem exer- } \\
\text { cícios. }\end{array}$ \\
\hline $\begin{array}{l}\text { Malagoni et al. }{ }^{14} \text {, } \\
\text { Itália }\end{array}$ & ECR não controlado & $\begin{array}{l}\mathrm{n}=289(210 \mathrm{H} \text { e } 79 \mathrm{M}) \\
\text { média de } 71 \pm 10,1 \text { anos }\end{array}$ & $\begin{array}{l}\text { Tolerância ao exercício: teste de } \\
\text { esteira com carga constante. } \\
\text { Qualidade de vida: com questio- } \\
\text { nário SF-36. }\end{array}$ & $\begin{array}{l}\text { Todos os participantes: cami- } \\
\text { nhada de } 10 \text { min } 2 x \text { por dia, } 6 \\
\text { dias por semana, durante } 2 \text { anos. } \\
\text { Posteriormente, a análise das } \\
\text { variáveis foi realizada entre os } \\
\text { participantes que tiveram alta } \\
\text { adesão, } 13 \pm 2 \text { meses }(\mathrm{G} 1) \text { com os } \\
\text { de baixa adesão, } 12 \pm 2 \text { meses (G2). }\end{array}$ \\
\hline $\begin{array}{l}\text { Collins et al. }{ }^{11}, \\
\text { EUA }\end{array}$ & ECR controlado & $\begin{array}{l}\mathrm{n}=103(89 \mathrm{H} \text { e } 14 \mathrm{M}) \\
\text { média de } 69,7 \pm 8,9 \text { anos }\end{array}$ & $\begin{array}{l}\text { Tolerância ao exercício: teste de } \\
\text { esteira com carga incremental, } \\
\text { carga constante (antes e após } 6 \text {, } \\
12 \text { e } 24 \text { semanas) e WIQ. Oxige- } \\
\text { nação tecidual: com NIRS. }\end{array}$ & $\begin{array}{l}\text { Exercício intervalado (baixa e } \\
\text { alta intensidade) } 3 x \text { por semana } \\
\text { durante } 24 \text { semanas. } \\
\text { G1: caminhada nórdica. } \\
\text { G2: caminhada tradicional. }\end{array}$ \\
\hline
\end{tabular}

\section{NA}

$\begin{array}{cc}\begin{array}{c}\text { Dziubek et al. }{ }^{15}, \\ \text { Polônia }\end{array} & \begin{array}{c}\text { Estudo transversal } \\ \text { do tipo caso-con- } \\ \text { trole }\end{array}\end{array}$

Lamberti et al. ${ }^{12}, \quad$ ECR controlado Itália

Akerman et al. ${ }^{13}$,

ECR controlado Nova Zelândia $\mathrm{n}=135(83 \mathrm{H}$ e 52 M), G1: 85 idosos com DAP e G2: 50 idosos sem DAP, média de $70,35 \pm 7,7$ anos

$\mathrm{n}=27(21 \mathrm{H}$ e $6 \mathrm{M})$, média de $67 \pm 7$ anos

Tolerância ao exercício: TC6, mais avaliação da força muscular dinâmica dos MMIl e pico de torque com dinamômetro isocinético

Tolerância ao exercício e claudicação: teste de esteira com carga constante e TC6.

Qualidade de vida: com questionário SF-36. Também avaliou o custo/efetividade do tratamento e adesão.

$\mathrm{n}=22(15 \mathrm{H} \mathrm{e} 7 \mathrm{M})$, média de $75,3 \pm 8,9$ anos

Tolerância ao exercício: teste de caminhada, TC6.

Temperatura interna durante atividade física: cápsula de telemetria.

Oxigenação e volume do sangue, função vascular: com a FMD, NIRS, VOP, pletismografia de oclusão venosa e reinalação de $\mathrm{CO}$.

Qualidade de vida: com questionário SF-36.

Também avaliou a aderência ao programa.

$\mathrm{CO}$ = monóxido de carbono; $\mathrm{ECR}$ = ensaio clínico randomizado; $\mathrm{FMD}$ = dilatação mediada por fluxo; $\mathrm{G} 1$ = grupo um; $\mathrm{G} 2$ = grupo dois; $\mathrm{H}=$ homens; $\mathrm{M}=$ mulheres; $\min =$ minutos; $\mathrm{MMII}=$ membros inferiores; $\mathrm{MMSS}=$ membros superiores; $\mathrm{n}=$ número de participantes; NA = não se aplica; NIRS = near-infrared spectroscopy (espectroscopia no infravermelho próximo); SF-36 = Medical Outcomes Short-Form Health Survey; TC6 = teste de caminhada de 6 minutos; $\mathrm{VO}_{2} \max =$ volume máximo de oxigênio; $\mathrm{VOP}$ = velocidade de onda de pulso; WIQ = Walking Impairment Questionnaire. 
Tabela 2. Nível de evidência, grau de recomendação e qualidade metodológica dos estudos selecionados.

\begin{tabular}{ccc}
\hline Autor & $\begin{array}{c}\text { Nível de evidência - grau } \\
\text { de recomendação }\end{array}$ & $\begin{array}{c}\text { Qualidade } \\
\text { metodológica }\end{array}$ \\
\hline Langbein et al. $^{9}$ & 1b-A & $6 / 10$ \\
Tew et al. ${ }^{10}$ & 1c-A & $3 / 10$ \\
${\text { Malagoni et al. }{ }^{14}}^{14}$ & 1b-A & $5 / 10$ \\
Collins et al. ${ }^{11}$ & 1b - A & $5 / 10$ \\
Dziubek et al. $^{15}$ & 1b-A & $5 / 10$ \\
Lamberti et al. $^{12}$ & 3b - B & NA \\
Akerman et al. ${ }^{13}$ & 1b - A & $6 / 10$ \\
\hline
\end{tabular}

$\mathrm{NA}=$ não se aplica.

Já a pesquisa de Collins et al. ${ }^{11}$ incluiu idosos com DAP que apresentavam índice tornozelo-braquial $\leq 0,90$ ou alguma evidência de vasos calcificados. Os participantes foram divididos em dois grupos: caminhada nórdica ou tradicional. Os autores relataram que ambos os grupos apresentaram melhora gradativa na oxigenação tecidual durante 24 semanas, porém não houve diferenças na função física ou na distância percorrida.

Entretanto, Langbein et al. ${ }^{9}$ mostraram que a caminhada nórdica melhorou significativamente $(\mathrm{p}<0,001)$ a tolerância ao exercício, verificada pelos testes de carga constante e incremental em esteira, e também a distância total percorrida $(\mathrm{p}<0,001)$, a velocidade da caminhada $(\mathrm{p}<0,02)$ e o $\mathrm{VO}_{2} \max (\mathrm{p}=0,01)$. Houve também redução dos níveis de percepção de dor logo após a aplicação do programa de treinamento.

Ainda utilizando o exercício de caminhada, Langbein et al. ${ }^{9}$ e Malagoni et al. ${ }^{14}$ avaliaram seus efeitos, porém na modalidade tradicional. Ambos retrataram aumento significativo na distância para claudicação inicial e absoluta, com acréscimo também na velocidade da marcha e no limiar da dor. Malagoni et al. ${ }^{14}$ ainda destacaram que os resultados foram mais significativos no grupo que melhor aderiu ao programa de tratamento.

Uma forma diferente de intervenção foi adotada por outras duas pesquisas. Tew et al. ${ }^{10}$ utilizaram o treino aeróbico de MMSS realizado em ergômetro e, curiosamente, relataram resultados satisfatórios no desempenho da caminhada. Esse achado foi atribuído, pelo menos em parte, ao aumento da oferta de oxigênio aos MMII. Ainda assim, houve melhora da cinética do $\mathrm{VO}_{2} \max$ (de 44,7 $\pm 10,4$ para $41,3 \pm 14,4$ segundos) e do tempo mínimo para saturação de oxigênio tecidual (de $268 \pm 305$ para $410 \pm 366$ segundos), que também aumentou significativamente $(p<0,05)$ durante a caminha em esteira.

Já Akerman et al. ${ }^{13}$ utilizaram imersões (a aproximadamente $39{ }^{\circ} \mathrm{C}$ ), exercícios resistidos e calistênicos, que resultaram no aumento da distância para claudicação absoluta de $350 \mathrm{~m}$ para $391 \mathrm{~m}(\mathrm{p}=0,006)$ e da claudicação inicial, que passou de $170 \mathrm{~m}$ para $213 \mathrm{~m}(\mathrm{p}<0,001)$. Não foram observadas alterações significativas em termos de volume sanguíneo, índice tornozelo-braquial, resultados funcionais ou níveis pressóricos; porém, a adesão e a tolerância à termoterapia foram excelentes, segundo os autores relataram.

No que se refere às contribuições da fisioterapia para o tratamento, apenas o estudo de Akerman et al. ${ }^{13}$ mencionou claramente o papel do fisioterapeuta. Os demais não relataram qual profissional ficou responsável por conduzir a intervenção.

\section{Impacto da DAP na QV de idosos e o papel da fisioterapia}

Apenas quatro pesquisas ${ }^{11-14}(57,14 \%)$ abordaram a efetividade da intervenção na QV dos idosos. O efeito dos protocolos propostos sobre esse desfecho foi variável. Os estudos realizados por Lamberti et al. ${ }^{12}$ e Malagoni et al. ${ }^{14}$ relataram que houve melhora na QV dos idosos, no que se refere aos domínios das dimensões física e emocional.

Por outro lado, Collins et al. ${ }^{11}$ e Akerman et al. ${ }^{13}$ não observaram diferenças significantes entre os grupos. Uma provável explicação para os resultados obtidos pode estar relacionada ao fato de que a melhora na QV desses pacientes não esteve necessariamente atrelada à intervenção proposta, mas sim à execução dos exercícios físicos ou à intervenção cirúrgica, fato que corrobora o postulado pela literatura.

Quanto ao domínio emocional, apenas o estudo de Akerman et al. ${ }^{13}$ apresentou redução de 35 pontos no escore, independentemente da intervenção. Todavia, observou-se que o domínio dor foi significativamente menor $(p=0,041)$, sendo que a pesquisa utilizou termoterapia por imersão associada à prática de exercícios de resistência e calistênicos. Não foram observadas outras alterações significativas na QV para os demais domínios do SF-36.

Em relação às dimensões física e mental, Collins et al. ${ }^{11}$, que utilizaram como abordagem terapêutica a caminhada nórdica, não observaram diferenças significativas entre os grupos intervenção e controle $(p=0,96$ para a dimensão física e $p=0,43$ para a dimensão mental). Informações mais detalhadas sobre os domínios do SF-36, como os valores exatos dos escores, não foram relatadas pelos autores.

Entre as pesquisas que adotaram a caminhada tradicional, Lamberti et al. ${ }^{12}$ destacaram que a adesão ao tratamento foi alta $(75 \%)$, repercutindo nos escores do SF-36, os quais melhoraram significativamente após a conclusão do programa de reabilitação, sendo menores no grupo de participantes com baixa adesão (25\%).

Os autores ainda acrescentam que não houve diferença significativa entre os grupos na dimensão física do questionário, e que somente o domínio capacidade funcional revelou um aumento significativo no grupo de participantes que foram submetidos 
a revascularização $(\mathrm{p}=0,041)$, mostrando que o tratamento intervencionista foi mais efetivo.

De maneira semelhante, Malagoni et al. ${ }^{14}$ mostraram um impacto positivo na QV dos pacientes em todos os domínios do SF-36, principalmente em relação às áreas de capacidade funcional (aumento de 18,9 pontos) e dor (aumento de 22,5 pontos). Pôde-se observar que todos os escores dos domínios do SF-36 melhoraram significativamente após a conclusão do programa de reabilitação, exibindo valores estatisticamente significativos $(\mathrm{p}<0,0001)$.

\section{CONCLUSÕES}

As intervenções terapêuticas avaliadas nos estudos contribuíram para melhora da tolerância ao exercício em idosos, com aumento da distância percorrida, da velocidade da marcha e da distância livre de sintomas, independente da modalidade empregada. Além disso, alguns estudos relataram que houve melhora na capacidade funcional máxima, medida por meio do $\mathrm{VO}_{2}$ max. Esses ganhos funcionais refletiram positivamente na $\mathrm{QV}$, com melhora nos escores totais e por domínio do questionário SF-36.

É importante ressaltar que não houve padronização dos protocolos utilizados, o que inviabiliza a identificação da superioridade de alguma intervenção em relação a outra. No entanto, a maioria dos tratamentos incluíram exercícios aeróbicos. Em suma, fica claro que as terapias apresentadas podem beneficiar idosos com DAP; porém, se faz necessária uma padronização dos protocolos de intervenção, de modo a favorecer a elaboração de diretrizes que auxiliem na prática clínica dos profissionais que atuam com esse perfil de pacientes.

\section{REFERÊNCIAS}

1. Alvim RO, Dias FAL, Oliveira CM, et al. Prevalence of peripheral artery disease and associated risk factors in a Brazilian rural population: the Baependi Heart Study. Int I Cardiovasc Sci. 2018;31:405-13. http://dx.doi.org/10.5935/2359-4802.20180031.

2. Corrêa UAC, Vidal AA, Gonçalves PEO, Sady ERR, Flumignan RLG, Cisneros LL. Fisioterapia intra-hospitalar para pacientes com isquemia crítica de membro inferior: consenso de especialistas. Fisioter Pesqui. 2019;26(2):151-7. http://dx.doi.org/10.1590/1809-2950/18006426022019.

3. Geiger MA, Guillaumon AT. Primary stenting for femoropopliteal peripheral arterial disease: analysis up to 24 months. J Vasc Bras. 2019;18:e20160104. PMid:31191625.

4. Mendez CB, Salum NC, Junkes C, Amante LN, Mendez CML. Mobile educational follow-up application for patients with peripheral arterial disease. Rev Lat Am Enfermagem. 2019;27:e3122. http:// dx.doi.org/10.1590/1518-8345.2693-3122. PMid:30698220.

5. Nunes $S$, Gouveia $C$. Afinal, não era preciso amputar! Um caso clínico. Rev Port Med Geral Fam. 2018;34(5):307-11. http://dx.doi. org/10.32385/rpmgf.v34i5.11834.

6. Cucato GG, Correia MA, Farah BQ, et al. Validation of a Brazilian Portuguese version of the walking estimated-limitation calculated by history (WELCH). Arq Bras Cardiol. 2016;106(1):49-55. http:// dx.doi.org/10.5935/abc.20160004. PMid:26647720.
7. Assis CS, Batista LC, Wolosker N, Zerati AE, Silva RCG. Functional independence measure in patients with intermittent claudication. Rev Esc Enferm USP. 2015;49(5):756-61. http://dx.doi.org/10.1590/ S0080-623420150000500007. PMid:26516744.

8. Aragão JA, Santos RM, Neves OMG, et al. Quality of life in patients with peripheral artery disease. J Vasc Bras. 2018;17(2):117-21. PMid:30377420.

9. Langbein WE, Collins EG, Orebaugh C, et al. Increasing exercise tolerance of persons limited by claudication pain using polestriding. J Vasc Surg. 2002;35(5):887-93. http://dx.doi.org/10.1067/ mva.2002.123756. PMid:12021703.

10. Tew G, Nawaz S, Zwierska I, Saxton JM. Limb-specific and crosstransfer effects of arm-crank exercise training in patients with symptomatic peripheral arterial disease. Clin Sci. 2009;117(12):40513. http://dx.doi.org/10.1042/CS20080688. PMid:19388883.

11. Collins EG, O'Connell S, McBurney C, et al. Comparison of walking with poles and traditional walking for peripheral arterial disease rehabilitation. J Cardiopulm Rehabil Prev. 2012;32(4):210-8. http:// dx.doi.org/10.1097/HCR.0b013e31825828f4. PMid:22595894.

12. Lamberti N, Malagoni AM, Ficarra V, et al. Structured homebased exercise versus invasive treatment: a mission impossible? A pilot randomized study in elderly patients with intermittent claudication. Angiology. 2016;67(8):772-80. http://dx.doi. org/10.1177/0003319715618481. PMid:26635335.

13. Akerman AP, Thomas KN, van Rij AM, Body ED, Alfadhel M, Cotter JD. Heat therapy vs. supervised exercise therapy for peripheral arterial disease: a 12-wk randomized, controlled trial. Am J Physiol Heart Circ Physiol. 2019;316(6):H1495. http://dx.doi.org/10.1152/ ajpheart.00151.2019. PMid:31002283.

14. Malagoni AM, Vagnoni E, Felisatti M, et al. Evaluation of patient compliance, quality of life impact and cost-effectiveness of a "test in-train out" exercise-based rehabilitation program for patients with intermittent claudication. Circ J. 2011;75(9):2128-34. http:// dx.doi.org/10.1253/circj.CJ-10-1311. PMid:21712607.

15. Dziubek W, Bulińska K, Stefańska M, et al. Peripheral arterial disease decreases muscle torque and functional walking capacity in elderly. Maturitas. 2015;81(4):480-6. http://dx.doi.org/10.1016/j. maturitas.2015.06.001. PMid:26119244.

Correspondência Jéssica Costa Leite Centro Universitário UNIFACISA Av. Senador Argemiro de Figueiredo, 1901 CEP 58411-020 - Campina Grande (PB), Brasil Tel.: (83) 2101-8877

E-mail: jessica.leite@maisunifacisa.com.br

Informações sobre os autores ALGL e JLS - Fisioterapeutas; Pós-graduandas em Fisioterapia em Terapia Intensiva Adulto e Infantil, Centro Universitário UNIFACISA.

JCL - Fisioterapeuta; Mestre em Fisioterapia; Doutoranda, Programa de pós-graduação em Fisioterapia, Universidade Federal do Rio Grande do Norte (UFRN); Professora, Curso de Fisioterapia, Centro Universitário UNIFACISA.

Contribuições dos autores Concepção e desenho do estudo: ALGL, JCL Análise e interpretação dos dados: ALGL, JLS, JCL Coleta de dados: ALGL, JLS Redação do artigo: ALGL, JLS Revisão crítica do texto: ALGL, JCL Aprovação final do artigo*: ALGL, JLS, JCL Análise estatística: N/A.

Responsabilidade geral pelo estudo: ALGL, JCL *Todos os autores leram e aprovaram a versão final submetida ao J Vasc Bras. 